\begin{tabular}{ccc}
\hline & $\begin{array}{r}\text { International Journal of Health Services } \\
\text { Research and Policy } \\
\text { www.dergipark.org.tr/ijhsrp }\end{array}$ \\
$\begin{array}{c}\text { INTERNATIONAL } \\
\text { ENGINEERING } \\
\text { SCIENCE AND } \\
\text { EDUCATION GROUP }\end{array}$ & e-ISSN: $2602-3482$ & IJHSRP \\
\hline ReWH
\end{tabular}

Review

\title{
NURSING INTERVENTIONS IN MANAGEMENT OF COVID-19-RELATED DELIRIUM IN INTENSIVE CARE UNITS: LITERATURE REVIEW
}

\author{
Emine KAPLAN SERIN ${ }^{* 1} \mathbb{1}$ Semra BÜLBÜLOĞLU $U^{2}$ (D)
}

\footnotetext{
${ }^{1}$ Department of Nursing, Faculty of Health Sciences, Gaziantep University, Gaziantep, Turkey

${ }^{2}$ Department of Nursing, Faculty of Health Sciences, Tokat Gaziosmanpasa University, Tokat, Turkey

* Corresponding author; emineserin@gantep.edu.tr
}

\begin{abstract}
The development of delirium is highly probable in COVID-19 patients, and its management is much more difficult than other diseases. Nurses have important roles in the management of delirium that may develop in COVID-19 patients. The aim of this paper was to review the literature regarding non-pharmacological interventions in intensive care units (ICUs) to prevent delirium in COVID-19 patients. The terms used in the literature search were 'intensive care' or 'critical care' including their abbreviations, as well as 'delirium,' 'intervention,' 'approach,' 'nursing' or 'non-pharmacologic' or 'treat' or 'management' or 'prevention' and 'COVID-19' with Turkish and English words. The literature was scanned using the keywords based on the Medical Subject Headings (MeSH) and Embase Tree (EMTREE) from the databases of Cochrane, CINAHL, PsyInfo PubMed, and EMBASE. A total of 63 studies were included in the review. In this paper, it was found that non-pharmacological interventions had an important place in delirium management; however, the transmission way of COVID-19 via droplets prevents many non-pharmacological attempts. First and foremost, among these interventions is the termination of the sedating drug, preventing isolation, ensuring that the patient spends time with visitors, and weaning from mechanical ventilation. In COVID-19 patients, the latest technology may be used to help prevent social isolation. Based on the findings of this literature review, we recommend a patient-centered approach in the management of delirium in COVID-19 patients and a revision of current strategies in accordance with the profiles of the patient and the disease. It is recommended to raise awareness on interventions for delirium syndrome that may cause problems for nurses regarding COVID-19.
\end{abstract}

Keywords: COVID-19, Delirium, Intensive Care Unit

Received: December 22, $2020 \quad$ Accepted: March 26, 2021

\section{Introduction}

The COVID-19 pandemic is an extremely dangerous threat for the world population, affecting all countries and requiring considerable financial and human resources [1]. The current literature highlights that it is dangerous especially for the elderly and individuals with chronic diseases [2]. It should not be ignored that many doctors, nurses and children and young people die due to the COVID-19 pandemic. 
The death toll of COVID-19 is expected to cause important problems for all sectors in the future. "Skilled labor", already scarce in an increasingly crowded world, may be safely predicted to be more expensive in the near future as a repercussion of the COVID-19 pandemic. Currently, the work of physicians and nurses is the first thing that comes to mind while speaking of skilled labor [3]. Delirium diagnosis, treatment, and care interventions require a multidisciplinary approach. Delirium diagnostic difficulties concern nurses as well as physicians. The lack of knowledge of physicians and nurse clinicians working in the ICU, lack of training on the topic, workload, and systemic problems may be listed in failure to diagnose delirium. This is because, when routine screening for delirium is not performed within the working system of the ICU, the patient is generally not evaluated in this respect [4]. The literature is constantly updated with the latest information on the negative effects of COVID-19 in the hospitalization process $[2,5,6]$.

Moreover, there are yet unknown but suspected secondary conditions of COVID-19, among which delirium is one of the most important ones [1].

Delirium is a transient neurocognitive syndrome associated with impaired cognitive functions, clouding of consciousness, inability to maintain attention, and unstable psychomotor activity [7,8]. Delirium often develops rapidly in a few days, worsens the clinical prognosis, and affects health status both in the short-term and long-term [8]. Before COVID-19, the mean incidence of delirium was 20$30 \%$ in clinics and $25-87 \%$ in Intensive Care Units (ICUs) [9].

Prior to COVID-19, it was reported that only $54 \%$ of health professionals involved in cases of delirium were using the correct terminology [10], and awareness of delirium and the capability to diagnose it were not adequate in many ICUs [11]. However, the development of delirium is related positively to morbidity, need for intensive care, length of time of mechanical ventilation and length of hospitalization, nosocomial infections, and mortality [7, 8, 12, 13]. Moreover, patients who experience delirium in the ICU are reported to continue having problems after their discharge and suffer functional deficiencies [12]. This underscores the potential long-term consequences of delirium after discharge caused by COVID-19. Delirium is quite common in the Intensive Care Units, and studies report that it is seen at rates varying between $25 \%$ and $87 \%$ [14-17]. In this context, it was noted that the rate of delirium in COVID-19 patients was 23\% [18]. Indeed, COVID-19 patients are directly at risk of delirium. In this context, the development of delirium is highly probable in COVID-19 patients, even inevitable, and its management is much more difficult than other diseases [1]. Therefore, it is very important that healthcare professionals acquire the awareness of delirium and the ability to detect sudden metabolic or physiological changes caused by COVID-19, as well as its diagnosis and treatment $[7,8$, 19]. The aim of this paper was to present a literature review regarding nonpharmacological strategies used in delirium management in patients treated for COVID-19 in the ICU.

\section{Material and Method}

The aim was to review the literature on non-pharmacological strategies used in the management of delirium in patients hospitalized for COVID-19 treatment in the ICU. The terms used in the literature search were 'intensive care' or 'critical care' including their abbreviations, as well as 'delirium,' 'intervention,' 'approach,' 'nursing' or 'non-pharmacologic' or 'treat' or 'management' or 'prevention' and 'COVID-19' with Turkish and English words. These keywords were determined based on the Medical Subject Headings (MeSH) and Embase Tree (EMTREE). Research articles published on the topic were 
found by scanning the databases of Cochrane, Cumulative Index to Nursing \& Allied Health Literature (CINAHL), PsycINFO, PubMed, and EMBASE between March and May 2020.

The inclusion criteria were publications in a refereed journal between 2000 and 2020, with an experimental or quasi-experimental design, full-text availability online, and being original and of quantitative nature. Figure 1 provides a summary of the search strategy results with a Flowchart of the Preferred Reporting Items for Systematic Reviews and Meta-Analyses (PRISMA) [20]. The screening returned a total of 136 studies. These were first examined by their titles, and 63 were excluded as they were not related to the scope of our study. Consequently, a total of 73 studies were included in the review.

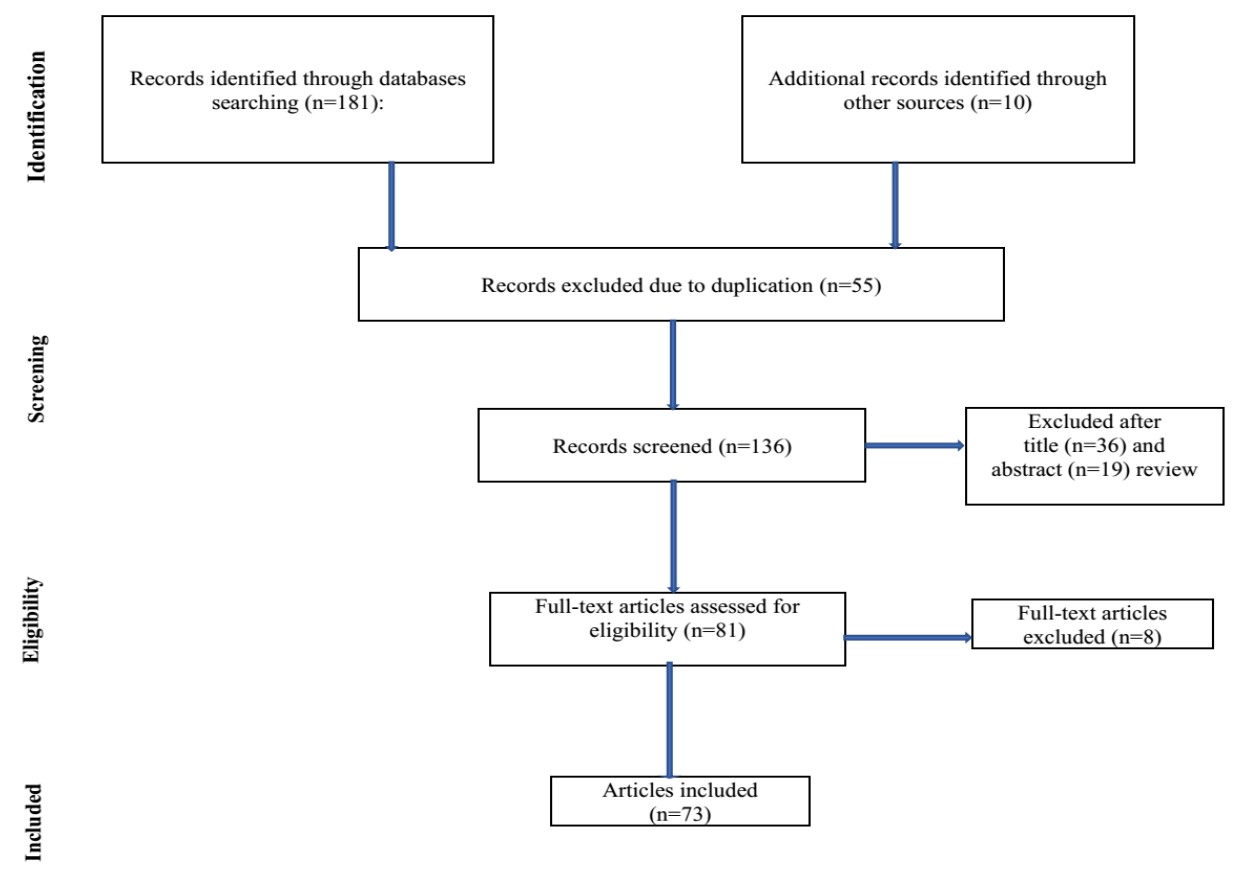

Figure 1. Flow diagram of study selection

\section{Results}

Delirium is assessed according to the Diagnostic and Statistical Manual of Mental Disorders (DSM-V) diagnostic criteria and delirium scales (Table 1). For a diagnosis of delirium, there should be 1) changes in consciousness and accompanying problems with concentration and attention, 2) disruptions in perception or cognitive functioning not related to dementia, 3) brief fluctuations during the day, 4) anamnesis, assumptions on a cause for dementia as a result of tests and examinations, and 5) exposure to toxicity should be determined [21]. More than one of these criteria are evaluated in the diagnosis of delirium. 


\section{Table 1. DSM-V Diagnostic Criteria}

A. Disruptions in attention: decreased ability to direct attention, focus, maintain and re-orient; decreased awareness.

B. Acute development: it usually develops within hours to several days, with a remarkable decrease in the initial level of attention and awareness.

C. Cognitive impairment: unresponsiveness to stimuli, weakened reflexes, memory problems, disorientation, impairment of language and eyesight, and compromised perception.

D. A neurocognitive disorder that develops as an extension of (A) and (C). A comatose condition and total unresponsiveness to stimuli may be observed.

E. Deterioration of the previous findings of physical examination or laboratory tests, such as a cognitive impairment which is a direct result of intoxication accompanied by another clinical diagnosis or exposure to a toxic agent, or which develops from multiple etiologies.

Pharmacological and non-pharmacological methods are used in delirium management. The side effects of medication used in the treatment of delirium include impaired heart rhythm, stroke in patients of dementia, restlessness, agitation, or sleepiness, and these medications may not always be useful in preventing delirium.

The United Kingdom National Institute for Health and Care Excellence (NICE) recommends short-term (maximum of one week) use of sedatives in agitated patients who are not communicative and show a risk to harm themselves or others [8,19]. Clinical practice guidelines also do not advise any pharmacological interventions in ICUs in the prevention and treatment of delirium [13]. Even though ICU delirium is considered to be a serious problem, the use of medication remains limited in clinical practice [9]. In consideration of these potential side effects, however, nonpharmacological interventions are needed in the prevention and treatment of delirium [8]. In a meta-analysis reviewing 14 studies, it was reported that non-pharmacological interventions reduced the frequency of delirium by $53 \%$ in patients hospitalized outside of ICUs and aged 65 and above [22].

\subsection{Non-Pharmacological Strategies in Delirium Management}

Multi-component interventions of care are needed in the diagnosis and management of delirium [8]. The diagnosis of delirium primarily includes identification and elimination of possible underlying problems, then the treatment, and prevention, and management of complications [8, 19]. Care approaches include cognitive, physical, psychological, and environmental interventions in accordance with clinical guidelines [8].

There is no specific treatment for delirium, but it is managed through multi-component nonpharmacological interventions that include effective communication, re-orientation, reducing environmental stimuli, cerebral hemodynamic recovery, cutting down daily sedation, early and frequent mobilization, exercise, hydration and sleep hygiene, family involvement, patient education and a holistic approach [7-9, 19, 23-25].

The most common practice is the ABCDE Bundle which involves Awakening and Breathing Coordination, Delirium prevention and management and Early physical mobility [5, 9, 12]. The multicomponent $\mathrm{ABCDE}$ Bundle reduces the incidence of delirium, as well as the length of ICU stay, dependency on mechanical ventilation and hospitalization and increases survival rates [12]. Bounds et 
al. (2016) reported the effects of ABCDE bundle application on delirium prevalence in intensive care patients in their study. In the study, after implementation of the ABCDE bundle, the prevalence of delirium decreased significantly (from $38 \%$ to $23 \%, \mathrm{P}=.01$ ), and the mean number of days of delirium were reduced significantly (from 3.8 to 1.72 days, $\mathrm{P}<$.001) [12]. The use of delirium prevention bundles is reported to reduce the frequency and length of delirium. These bundles consist of oxygen therapy, fluid-electrolyte balance, preventing dehydration, controlling pain and agitation, avoiding polypharmacy, regulating bladder and intestinal functions, adequate nutrition, regulating the environment and treating cognitive impairment, as well as problems with mobility, eyesight, and hearing $[7,9,13,25-27]$.

\subsection{Regulating the Environment:}

Environmental factors that trigger delirium include noise, constant light, odors, interventional or non-interventional procedures, sensory overload, insufficient cognitive and verbal stimuli, social isolation, inactivity, sleep disturbances, lack of humidity, heat, and windowless interiors [13]. Iatrogenic factors include the application of chest, nasogastric or endotracheal tubes, bladder catheterization, mechanical ventilation, invasive monitoring, and untreated urinary and stool retention [13]. Noise heightens adrenal and cardiovascular stimulation and suppresses the immune system [13].

Regulations of the environment should therefore involve lighting and noise management, keeping a calendar and clock, explaining the equipment required in the environment to the patient, controlling confusing or overwhelming stimuli, effective communication with the patient, daily re-orientation to persons, space and time, and providing a good environment for sleep to maintain the circadian rhythm $[8,13]$. Adding a radio or television, or hanging pictures of relatives near the bed in the unit may help maintain the patient's orientation [7].

Moreover, it is recommended to minimize physical restraints and noise, prevent malnutrition, conceal tubes and catheters that are used, ensure adequate lighting and minimize noise, control pain and monitor the medications administered for risk of triggering delirium $[8,13]$. The side rails of the bed should be raised for patient safety [8]. The patient in delirium should be closely monitored, using alarm, sound, and video camera equipment where necessary [13].

The recommended room temperature is between 21.1 and $23.8^{\circ} \mathrm{C}$, and the noise level should not be above 40 decibels in the daytime and 35 decibels at night $[7,13]$. These interventions to enhance patient comfort and relaxation are both low-risk and inexpensive [26].

\subsection{Additional Points of Patient Care}

Healthcare professionals should avoid procedures that are not essential or that only disrupt the patient especially in hours of sleep. In fact, they should minimize all care interventions, keep only a few lights on at night, and take noise-reducing measures, such as giving the patient washable earplugs or turning off device alarms, where possible $[7,12,26]$. Day and night may be explained from an appropriate distance showing the patient a window looking outside [13]. Where patients have impaired hearing or eyesight, these problems should be addressed in order to avoid the worsening of delirium and keep it under control [12]. Ensuring sleep hygiene and day and night cycles, use of night lamps, providing the patient with appropriate hearing aid or eyeglasses are other recommendations [8, 9, 25].

It should be remembered that lack of stimuli is likely to increase hallucinations in the patient. ${ }^{7}$ Interventions that improve the state of consciousness should not be forgotten, including providing the 
patient with suitable cognitive and therapeutic stimuli [12]. However, the stimuli should not be often or too many and confusing. Patients who develop delirium may be frightened and defensive. When they cannot express their feelings and thoughts verbally, they may seem anxious, restless, or stressed. Therefore, it is imperative that healthcare professionals need to gain the trust of the patient. Talking to the patient and discussing daily events and news with them are helpful in preventing delirium through re-orientation of the patient [8]. Moreover, visiting relatives in the ICU should be warned that the patient might be in delirium and the reasons [12] should be explained, whereas visitation times should also be extended [7, 9, 25, 27]. The religious (religious wellbeing) dimension in spiritual wellbeing may be interpreted as having a spiritual connection with God or a higher power [28]. The existential dimension is a mental and social element and shows who it is, what it does why, where it belongs [28]. The religious dimension leads us to God, and the existential dimension directs us to the environment and other people beyond ourselves. It is very important that intensive care nurses provide spiritual care. In situations where there are an impasse and conflict in spiritual wellbeing, the person may experience painful feelings such as loneliness, depression, and loss, and they may question the meaning of life [30, 31]. This situation requires spiritual care, social and psychological support [28, 29]. Spiritual wellbeing and care initiatives increase life expectancy, energy, and motivation [30]. They provide people with hope and thus strengthen their adaptation to disease conditions [32].

It is important to wake up patients once a day or at certain intervals during the day and conduct spontaneous breathing trials [12], test the level of neurological functions, and orient the patient. Having two patients with delirium share the same room should be avoided [13]. It is also needed to ensure the safety of the patient and inform the family members about all procedures [13].

The patient's personality, coping strategies, existing psychological problems, and levels of fear, panic, and anxiety are among the emotional factors that may lay the ground for delirium [13]. Repetition of a certain thought in delirium may aggravate the situation causing agitation; therefore, distracting the attention is recommended. The attention span of patients in delirium is short, and drawing attention with varying stimulation (like music or visuals) may help detach them from thoughts that cause agitation [13]. Moreover, dexmedetomidine derivatives should be preferred over derivatives of benzodiazepine in agitated patients [12]. It should be remembered that restraining always increases agitation [13]. If the patient has substance abuse problems, specific psychological support should be provided [13].

In their study, Simon et al. suggested using a combination of several measures that may be less effective or not be effective alone but decrease delirium incidence in ICU patients when used in a multicomponent format, such as light therapy and regulating the circadian rhythm [33].

Furthermore, Chevillon et al. reported that individual preoperative training of increasing the patient's knowledge before pulmonary thrombo-endarterectomy helped decrease the time the patient had to be kept on mechanical ventilation by increasing their knowledge [34, 35].

\subsection{Mobilization Exercises}

In the literature, there are different results regarding early and frequent mobilization exercises and the mechanism by which they work $[8,26]$. However, the combined use of non-pharmacological interventions is supported. ${ }^{7}$ Mobilization of patients by way of bed exercises ${ }^{24}$ within the first 48 hours after admission to the ICU is recommended to prevent delirium $[8,12,19]$.

Mobilization requires teamwork, and patient fitness needs to be assessed before getting started with exercises. In cases where exercise may have side effects, such as deep vein thrombosis, 
hematochezia, or severe cardiac ischemia, the healthcare team should consider if indicated and decide on the amount and kind of mobilization. Mobilization must be immediately terminated in case of disruption in the patient's breathing pattern or hemodynamic imbalance. Exercise is recommended for at least 20 minutes a day [8]. Exercise also reduces the risk of falling [12].

In their study to identify the effects of exercise in preventing delirium and shortening its duration in elderly ICU patients, Karadaş and Özdemir (2016) found no significant relationship even though the incidence and duration of delirium decreased 2.5 times compared to the control group [36]. Functional and cognitive impairments are adverse situations that require rehabilitation. With early physical and cognitive rehabilitation, the duration of delirium is shortened, and the underlying problems must be corrected simultaneously. In the long term, the effects of early physical and cognitive therapy should be evaluated $[8,9,22,25]$. The few studies available have indicated that rehabilitation of cognitive impairment is possible, but new studies are needed to determine its effectiveness. However, rehabilitation of functional disability and correction of cognitive impairment are important in reducing delirium and associated morbidity.

\subsection{Relationship of COVID-19 to Delirium}

\subsubsection{Delirium in COVID-19 Patients}

Studies show that $40-80 \%$ of patients hospitalized in the ICU experience delirium [4, 9, 37-45]. It is recorded that delirium develops in $60-80 \%$ of patients connected to mechanical ventilation and 40 $60 \%$ in those not connected, in $14-47 \%$ of elderly patients, in $30 \%$ of patients after intraabdominal surgery, in $30-67 \%$ of patients after heart surgery, in $40-50 \%$ of patients after orthopedic surgery, and in $26-44 \%$ of end-stage cancer patients in hospital [1, 4, 25, 42-47].

Investigation of the reasons why COVID-19 increases the incidence of delirium in the ICU points at risk factors such as the increased use of sedating medication together with mechanical ventilation, hallucinations caused by hydroxychloroquine, vasopressor shock, and use of mechanical ventilation by intubation $[1,25,46]$. High doses of sedatives in intubation and reintubation to prevent cough which increases the risk of spreading the virus through droplets to the healthcare team are other conditions that increase ICU delirium [1].

It is thought that there is a direct relationship between cytokine levels and delirium. The serum levels of interleukin (IL)-6 and IL-8, which are pro-inflammatory cytokines, have been shown to increase the incidence of delirium in elderly patients with hip fracture $[47,48]$.

Lower levels of the insulin-like growth factor (Insulin-like Growth Factor, IGF)-1 and lower levels of circulating IL-1RA are associated with delirium [49]. In addition to these, decreased serum Interferon Gamma (IFN- $\gamma$ ) is detected in elderly medical patients with delirium [50].

COVID-19 patients are thought to experience a significant increase in the level of virus-induced cytokines [51]. Reducing and treating hyper-inflammation is an important requirement with the use of validated treatments to reduce high mortality levels [51-54]. There is important evidence linking high IL-6 and IL-8 levels to delirium. A study with elderly patients hospitalized in acute conditions and who developed delirium found significantly high levels of IL-6 and IL-8 [47, 48]. A cytokine is a type of glycoprotein (which contains oligosaccharide chains-glycans covalently attached to amino acid sidechains) essentially synthesized by monocytes and macrophages, and in certain conditions, Tlymphocytes, neutrophils, mast cells, fibroblasts, and endothelial cells also contribute to its production. 
Cytokine is the first component that can be detected in the blood following tissue damage or physical stress, and it has important effects in the onset and continuation of inflammation in many autoimmune diseases [55].

The Tumor Necrosis Factor (TNF)- $\alpha$ is one of the pro-inflammatory cytokines responsible for inflammation, immunity, and cellular homeostasis. TNF- $\alpha$ plays a critical role also in tumor proliferation, migration, invasion, and angiogenesis. These cytokines may also be associated with the pathophysiology of delirium [56, 57]. Additionally, TNF- $\alpha$ may also mediate neurodegeneration by inhibiting the activity of IGF-1, a neuroprotective peptide. Peripheral TNF- $\alpha$, induced by any systemic inflammatory process, plays an important role in microglial activation and the release of more cytokines that have a detrimental effect on neuronal functions in brain tissue [56].

\subsubsection{Treatment of COVID-19}

The current treatment of COVID-19 is supportive therapy. Acute respiratory distress syndrome (ARDS) and respiratory failure are the leading causes of mortality. More than $70 \%$ of patients who die have been found to have ARDS [3,4]. In addition to respiratory system problems, multiorgan failure and sepsis are responsible for $60 \%$ of the mortalities [58]. The frequency of hemophagocytic lymphohistiocytosis is relatively high in adults, and it is common in $50 \%$ of viral infections that cannot be cured [58]. HLH is an important disease in which the immune system is overstimulated and causes multiorgan failure at advanced stages. Consequently, HLH is found in 3.7 to $4.3 \%$ of the cases of sepsisinduced by COVID-19 [59]. Its symptoms include fever, cytopenia, and hyperferritinemia [51].

Approximately 50\% of patients with pulmonary involvement experience HLH [60]. In severe cases of COVID-19, the gravity of the illness is characterized by an increase in the levels of cytokines, IL-2, IL-7, granulocytic colony-stimulating factor, interferon- $\gamma$ inducing protein 10 , monocyte chemoattractant protein ${ }^{1}$, macrophage inflammatory protein $1-\alpha$ and TNF- $\alpha$ [61]. A recent retrospective multi-center study found a correlation between lethal prognosis and hyper-inflammation. In a retrospective study, it was found that patients who died eventually had higher ferritin and IL-6 levels than those who survived [53].

As with previous pandemics, corticosteroids are not routinely recommended and are supposed to exacerbate pulmonary damage associated with COVID-19 [62]. Notwithstanding, randomized controlled studies on patients with sepsis hyper inflammation showed that IL-1 blockade (anakinra) was beneficial and did not increase adverse events [63]. Consequently, therapeutic options advised for COVID-19 include steroids, intravenous immunoglobulin, selective cytokine blockade (e.g., anakinra or tocilizumab), and Janus kinase inhibitors [51,63].

SARS-CoV-2 affects the central nervous system through peripheral nerves. It is thought to affect respiration and sense of smell through its action on the hypothalamus and brainstem through the nose. SARS-CoVs enter human host cells via the cellular receptor of the angiotensin-converting enzyme (ACE2). The possible brain entry paths of SARS-CoV-2 also include direct intranasal access through olfactory nerves (with anosmia as an early symptom) or indirect access through hematogenous or lymphatic spread passing the blood-brain barrier. Coronavirus causes brain damage, disrupting the renin-angiotensin system. ACE is the main component of the renin-angiotensin system and is localized in the endothelium of the cerebral vasculature. The use of ACE inhibitors to prevent hypertension reduces cognitive dysfunction. The inflammatory response of the central nervous system to viral infection may cause a poor neurological outcome and delirium. Neutrophils and monocytes penetrate 
into the central nervous system in the case of infection. Immune cells infiltrate the brain, and macrophages and microglia play a role in the destruction of myelin. As a result, neurological disorders may lead to delirium [64].

\subsubsection{Delirium Care in COVID-19}

It has been determined that the rate of delirium in patients connected to a mechanical ventilator is $70-75 \%$ [64]. A longer stay on a mechanical ventilator increases the risk of delirium, increases the length of hospital stay, and increases the cost. In the future, patients are more likely to suffer from dementia $[65,66]$. The risk of delirium may be prevented by $50 \%$ with less sedation, earlier mobilization, and early extubation (weaning) [64].

The use of the ABCDEF care package may have significant benefits [67]. This is because it includes attempts to reduce the sadness, isolation, fear, and helplessness caused by staying in intensive care, and it creates awareness on life. It is an accurate and necessary practice for clinicians regarding the ABCDEF care bundle to use reminders.

Routine ICU care is already performed in patients with COVID-19. It is worrying that a reliable measurement instrument is not routinely used to diagnose delirium. Therefore, awareness of the ICU team members on delirium detection and management should be developed in both physicians and nurses, and their delirium diagnosis should be checked [68].

The largest obstacle in detecting delirium in the ICU is the use of sedation. Sedation is inevitable in hyperactive delirium. This makes it difficult to provide space-time orientation in patients or may trigger delirium. For this reason, sedation and termination of the mechanical ventilator are very important. However, effective pain management should be done first. This is because severe pain not only triggers delirium and but also requires high doses of sedation. The high dose and long-term use of sedation cause the patient to be immobile unnecessarily and prevents mobilization.

It has been determined that delirium seen in COVID-19 patients increases cost, mortality, and morbidity. To put it more clearly, delirium directly affects survival in COVID-19 patients [64]. In literature reviews, ineffective and insufficient pain management, gastrointestinal problems, urinary retention, nosocomial sepsis, and insufficient oxygenation has been identified as delirium triggers in COVID-19 patients $[64,69]$. Delirium risks should be minimized in the ICU.

The preferred prone position in COVID-19 patients requires more sedation [70]. This may increase the delirium in delirium patients. Additionally, it is ideal to choose drugs that have low toxicity and will not cause neurotoxicity in COVID-19 drug administration, because neurotoxicity will increase the risk of delirium.

\section{Discussion}

COVID-19 claimed tens of thousands of lives worldwide. As of yet, it is not possible to predict any future health problems that might befall the survivors. The disruptions COVID-19 causes in the blood-brain barrier and cerebral circulation, physiological changes due to hyperinflammation, and environmental stressors in ICUs lay the groundwork for delirium as a secondary condition. However, before the onset of the COVID-19 outbreak, delirium management in ICUs was far from being optimal due to insufficient financial and human resources $[5,9,12]$. Today, COVID-19 further increases the workload of healthcare professionals. As a result, there is a real need for new financial and human resources for the management of delirium caused by COVID-19 and other problems in ICUs [64]. 
Some non-pharmacological methods in delirium management may not be effective or feasible in COVID-19 patients due to the disease-specific difficulties of the condition. The physical distance between patients is of crucial importance as the primary transmission path of COVID-19 is the respiratory tract. Special clothing of healthcare professionals may make it difficult for the patient to recognize them. This, in turn, may cause patients to experience more anxiety and isolation.

Furthermore, deep sedation of COVID-19 patients to inhibit the cough reflex [1] may cause delirium [25]. In COVID-19 patients, prevention of transmission through droplets is vitally important and can only be achieved through deep sedation. Patients surviving COVID-19 may experience withdrawal signs related to the sedation drugs used including those used for delirium. Another risk factor in addition to deep sedation is long-term mechanical ventilation in ICU stays $[1,25,46]$.

Apart from the problems caused by the drugs used in the treatment of COVID-19, the virus itself is thought to cause pulmonary, renal, hepatic, psychiatric, neurological, and cardiovascular problems [71]. Psychiatric and neurological problems including headache, unconsciousness, muscular coordination disorder, acute cerebrovascular disease, confusion, and convulsive seizures are likely to be seen within 2-3 weeks after catching the novel coronavirus [71, 72].

Mobilization of patients receiving deep sedation and mechanical ventilation is an important challenge. It certainly does not allow active participation in bed exercises and physiotherapy interventions. Communication with patients, conversations, and close contact with family and friends is an effective intervention in the prevention of delirium in the ICU. However, the visitation restrictions inevitably imposed on COVID-19 patients because of the dangers of close physical contact render this strategy impossible to implement. For the same reason, healthcare professionals cannot spend much time with the patient or make lengthy observations.

In literature reviews, it has been reported that after the acute phase of COVID-19, viruses remain in the central nervous system (CNS) and subsequently lead to post-infectious neurological complications [73]. The degree and severity of the COVID-19 disease process are significantly associated with the development of psychiatric and neurological problems. Severe systemic inflammatory responses are likely to induce these problems. The psycho-neurological vulnerability of individuals with advanced age, weak immune system, comorbidity, or chronic disease increases [44]. Even if this group of patients survives COVID-19, even minor injuries such as urinary tract infection or pneumonia may trigger an acute confusion state, delirium, and encephalopathy, as there will now be a predisposition [44]. In the literature, the D-dimer level in the blood of patients with at least one neurological symptom was found to be significantly higher $(\mathrm{p}<0.05$ ) than patients without neurological symptoms. IL-6 levels were found to be significantly higher in patients with a headache than those without $(\mathrm{p}<0.05)$. The creatine kinase (CK) level was found to be significantly higher in patients with muscle pain in comparison to those without muscle pain ( $\mathrm{p}<0.05$ ). Increasing awareness of neurological and psychiatric problems caused by COVID-19 is of great importance for the management, prevention, and treatment of these problems.

Delirium is a potential problem that COVID-19 patients may experience during COVID-19 treatments, and its management is crucially important. To this end, the latest technology may be used. Video conversations using smartphones or teleconferences with headsets to support and enhance patient's interaction with family and friends help prevent social isolation [64]. 


\section{Conclusion}

Delirium is a significant mental condition that may be frequently observed in COVID-19 and other ICU patients which increases the rates of morbidity and mortality. Delirium might and is likely to continue after discharge from the hospital. The risk, management, and treatment of delirium are all multifactorial. Effective methods for prevention and treatment include respiratory coordination, early discontinuation of sedative medication or continuation with low dosages, monitoring and management, exercise, and early mobilization.

Non-pharmacological interventions may be performed with a trained interdisciplinary team to keep agitation, pain, and delirium under control in ICUs, through fluid and nutrition support, reorientation, appropriate lighting, noise prevention, mobilization, reduction of evening stimuli, regulating the environment, and regulating the circadian rhythm with sleep protocols. However, management of delirium by healthcare professionals requires arrangements where non-pharmacological methods may be used in COVID-19 patients.

Funding: This study did not receive any specific grant from funding agencies in the public, commercial or not-for-profit sectors.

\section{Conflicts of interest: None.}

The compliance to Research and Publication Ethics: This work was carried out by obeying research and ethics rules.

\section{References}

[1] LaHue, S.C., et al.," Collaborative Delirium Prevention in the Age of COVID- 19" Journal of the American Geriatrics Society, 68(5), 947-949, 2020.

[2] Wu, Z., McGoogan, J.M., 'Characteristics of and Important Lessons from the Coronavirus Disease 2019 (COVID-19) Outbreak in China: Summary of a Report of 72314 Cases from the Chinese Center for Disease Control and Prevention', JAMA, 323(13), 1239-1242, 2020.

[3] World Health Organization, 'Protocol for assessment of potential risk factors for coronavirus disease 2019 (COVID-19) among health workers in a health care setting, 2020", https://apps.who.int/iris/handle/10665/332071.

[4] Bülbüloğlu, S., Delirium Definition and Subgroups, in: Acute Brain Disfunction and Care (Ed. S. Bülbüloğlu), Çukurova Nobel Medicine Publisher, Antalya, Turkey, 1-8, 2020.

[5] Novel Coronavirus Pneumonia Emergency Response Epidemiology Team, 'Vital surveillances: the epidemiological characteristics of an outbreak of 2019 novel coronavirus diseases (COVID19)-China, 2020”, China CDC Weekly, 2(x), 1-10, 2020,

[6] Battegay, M., et al., '2019-Novel coronavirus (2019-nCoV): estimating the case fatality rate: a word of caution", SwissMedWkly, 150, w20203, 2020.

[7] Dilek, F., et al., 'Most Seen Problems and Nursing Care in Geriatric Patients Receiving Intensive Care Treatment: Review", Intensive Care J Nurs, 9(1), 29-35, 2015. 
[8] Karadaş, C., 'Non-pharmacological approaches and nursing responsibilities in delirium management', J Psychiatr Nurs, 10(2), 137-142, 2019.

[9] Kang, J., et al., 'Effect of nonpharmacological interventions for the prevention of delirium in the intensive care unit: A systematic review and meta-analysis"', J Crit Care, 48, 372-384, 2018.

[10] Morandi, A., et al., 'Understanding international differences in terminology for delirium and other types of acute brain dysfunction in critically ill patients', Intensive Care Med, 34(10), 1907-1915, 2008.

[11] Spronk, P.E., et al., 'Occurrence of delirium is severely underestimated in the ICU during daily care', Intensive Care Med, 35(7), 1276-1280, 2009.

[12] Bounds, M., et al., 'Effect of ABCDE bundle implementation on prevalence of delirium in intensive care unit patients", Am J Crit Care, 25(6), 535-44, 2016.

[13] Özdemir, L., 'The management of delirium in intensive care patients and the responsibilities of nurses', Hacettepe University J Nurs, 1(1), 90-98, 2015.

[14] Skrobik, Y., Ahern, S., Leblanc, M., Marquis, F., Awissi, D.K., Kavanagh, B.P., "Protocolized intensive care unit management of analgesia, sedation, and delirium improves analgesia and subsyndromal delirium rates", Anesth Analg, 111, 451-463, 2010.

[15] Pisani, M.A., Kong, S.Y., Kasl, S.V., Murphy, T.E, Araujo, K.L., Van Ness, P.H., 'DDays of delirium are associated with 1-year mortality in an older intensive care unit population", Am J Respir Crit Care Med, 180, 1092-1097, 2009.

[16] Ely, E.W., Shintani, A., Truman, B., et al., 'Delirium as a predictor of mortality in mechanically ventilated patients in the intensive care unit", JAMA, 291(14), 1753-1762, 2004.

[17] McNicoll, L., Pisani, M.A., Zhang, Y., Ely, E.W., Siegel, M.D., Inouye, S.K.,’’ Delirium in the intensive care unit: occurrence and clinical course in older patients", J Am Geriatr Soc, 51(5), 591-598, 2003.

[18] Paterson, R.W., et al.," The emerging spectrum of COVID-19 neurology: clinical, radiological and laboratory findings", Brain, 143(10), 3104-3120, 2020

[19] NICE Guidance, Delirium: prevention, diagnosis, and management, 2010, www.nice.org.uk/guidance/cg103

[20] Moher, D., Liberati, A., Tetzlaff, J., Altman, D.G., '’PRISMA group preferred reporting items for systematic reviews and meta-analyses: the PRISMA statement. Ann Intern Med, 151(4), 264-9, 2009.

[21] American Psychiatric Association, Diagnostic and Statistical Manual of Mental Disorders: DSM-5-5th ed. Arlington, VA, American Psychiatric Publishing, 2013.

[22] Hshieh, T.T., et al. 'Effectiveness of multicomponent nonpharmacological delirium interventions: a meta-analysis," JAMA Intern Med, 175(4), 512-20, 2015. 
[23] Stuck, A., et al., "Preventing intensive care unit delirium: a patient-centered approach to reducing sleep disruption", Dimens Crit Care Nurs, 30(6), 315-320, 2011.

[24] Warshaw, G., Mechlin, M.," Prevention and management of postoperative delirium', Int Anesthesiol Clin, 47(4), 137-49, 2009.

[25] Devlin, J.W., et al.," Clinical practice guidelines for the prevention and management of pain, agitation/sedation, delirium, immobility, and sleep disruption in adult patients in the ICU', Crit Care Med, 46(9), 825-73, 2018.

[26] Brummel, N.E., Girard, T.D., 'Preventing delirium in the intensive care unit' Crit Care Clin, 29(1), 51-65, 2013.

[27] Köksal, G.M., 'Delirium', http://turkishjic.org/archives/archive-detail/article preview/deliryum/4266.

[28] Fathi, M., Hamzepour, H., Gammon, J., Roshani, D., Valiee, S., ' Hope and spırıtual well-being in iranian patients undergoing chemotherapy. Journal of Evolution of Medical and Dental Sciences-Jemds, 7(27), 3106-3111, 2018.

[29] Fatemi, S.N., Rezaei, M., Givari, A., et al., 'Prayer and spiritual well-being in cancer patients', J Iran Institute Health Sci Res, 5(4), 295-303, 2006.

[30] Mohammadi, S., Z., Tajvidi, M., 'Relationship between spiritual well-being with hopelessness and social skills in Beta-thalassemia major adolescents", Modern Care Journal, 8(3), 116-24, 2011.

[31] Neathery, M., Taylor, E. J., He, Z., 'Perceived barriers to providing spiritual care among psychiatric mental health nurses", Archives of Psychiatric Nursing, 34(6), 572-579, 2020.

[32] Nelson, C.J., Rosenfeld, B., Breitbart, W., et al., 'S Spirituality, religion and depression in the terminally ill”, Psychosomatics, 43(3), 213-20, 2002.

[33] Simons, K.S., et al. 'Dynamic light application therapy to reduce the incidence and duration of delirium in intensive-care patients: a randomised controlled trial' 'Lancet Respir Med, 4(3), 194-202, 2016.

[34] Chevillon, C., et al., 'Preoperative Education on Postoperative Delirium, Anxiety, and Knowledge in Pulmonary Thromboendarterectomy Patients', Am J Crit Care, 24(2), 164-71, 2015.

[35] Oh, E.S., et al., 'Delirium in Older Persons: Advances in Diagnosis and Treatment', JAMA, 318(12), 1161-74, 2017.

[36] Karadas, C., Ozdemir, L.," The effect of range of motion exercises on delirium prevention among patients aged 65 and over in intensive care units", Geriatr Nurs NYN, 37(3), 180-5, 2016.

[37] Tomichek, J.E., et al." Antipsychotic prescribing patterns during and after critical illness: a prospective cohort study," Crit Care, 20(1), 378, 2016. 
[38] Barr, J., et al., ' 'Clinical practice guidelines for the management of pain, agitation, and delirium in adult patients in the intensive care unit', Crit Care Med, 41(1), 263-306, 2013.

[39] Vallabhajosyula, S., et al., 'Role of statins in delirium prevention in critical ill and cardiac surgery patients: a systematic review and meta-analysis', J Crit Care, 37,189-96, 2017.

[40] Van den Boogaard M, et al., ' Effect of haloperidol on survival among critically ill adults with a high risk of delirium: the REDUCE randomized clinical trial', JAMA, 319(7), 680-90, 2018.

[41] Avidan, M.S., et al., ,' Intraoperative ketamine for prevention of postoperative delirium or pain after major surgery in older adults: an international, multicentre, double-blind, randomised clinical trial', Lancet, 390(10091), 267-75, 2017.

[42] Schuurmans, M.J., et al., 'Early recognition of delirium: Review of the literature', J Clin Nurs, 10, 721-729, 2001.

[43] Hipp, D.M., 'Pharmacological and Non-pharmacological Management of delirium in critically ill patients", Neurotherapeutics, 9(1), 158-175, 2012.

[44] Kaplan Serin, E., Strategies to Reduce and Prevent Delirium in the ICU, in Acute Brain Disfunction and Care, (Ed. S. Bülbüloğlu), Çukurova Nobel Medicine Publisher, Antalya, Turkey, 139-146, 2020.

[45] Maldonado, J.R., 'Delirium in the Acute Care Setting: Characteristics, Diagnosis and Treatment' Crit Care Clin, 24, 657-722, 2008.

[46] Inouye, S.K., et al., “' Delirium in elderly people’, Lancet, 383(9920), 911-922, 2014.

[47] van Munster, B.C., et al., " Time- course of cytokines during delirium in elderly patients with hip fractures", Journal of the American Geriatrics Society, 56(9), 1704-1709, 2008.

[48] de Rooij, S.E., et al., 'Cytokines and acute phase response in delirium', Journal of psychosomatic research, 62(5), 521-525, 2007.

[49] Adamis, D., et al., 'Cytokines and IGF-I in delirious and non-delirious acutely ill older medical inpatients"', Age and aging, 38(3), 326-332, 2009.

[50] Wilson, K., et al., 'Plasma insulin growth factor-1 and incident delirium in older people', International Journal of Geriatric Psychiatry: A journal of the psychiatry of late life and allied sciences, 20(2), 154-159, 2005.

[51] Mehta, P., et al., 'COVID-19: consider cytokine storm syndromes and immunosuppression', The Lancet, 395(10229), 1033-1034, 2020.

[52] WHO., 'Coronavirus disease 2019 (COVID-19) situation report - 52", https://www.who.int/docs/default-source/ coronaviruse/ 20200312-sitrep-52-covid-19. pdf?sfvrsn=e2bfc9c0_2.

[53] Ruan, Q., et al., "Clinical predictors of mortality due to COVID-19 based on an analysis of data of 150 patients from Wuhan, China', Intensive Care Med, 46(5), 846-848, 2020. 
[54] Lemstra, A.W., et al., 'Pre- operative inflammatory markers and the risk of postoperative delirium in elderly patients", International Journal of Geriatric Psychiatry: A journal of the psychiatry of late life and allied sciences, 23(9), 943-948, 2008.

[55] Vilcek, J., 'First demonstration of the role of TNF in the pathogenesis of disease", The Journal of Immunology, 181(1), 5-6, 2008.

[56] van Gool, W.A., et al., 'Systemic infection and delirium: when cytokines and acetylcholine collide", The Lancet, 375(9716), 773-775, 2010.

[57] Cerejeira, J., et al., 'The neuroinflammatory hypothesis of delirium', Acta neuropathologica, 119(6), 737-754, 2010.

[58] Ramos-Casals, M., et al., 'Adult haemophagocytic syndrome', Lancet, 383, 1503-1516, 2014.

[59] Karakike, E., Giamarellos-Bourboulis, E.J., 'Macrophage activation-like syndrome: a distinct entity leading to early death in sepsis", Front Immunol, 10, 55, 2019.

[60] Seguin, A., et al., 'Pulmonary involvement in patients with hemophagocytic lymphohistiocytosis", Chest, 149, 1294-301, 2016.

[61] Huang, C., et al., 'Clinical features of patients infected with 2019 novel coronavirus in Wuhan, China", Lancet, 395, 497-506, 2020.

[62] Russell, C.D., et al., 'Clinical evidence does not support corticosteroid treatment for 2019-nCoV lung injury", Lancet, 395, 473-475, 2020.

[63] Shakoory, B., et al., 'Interleukin-1 receptor blockade is associated with reduced mortality in sepsis patients with features of macrophage activation syndrome: reanalysis of a prior phase iii trial', Crit Care Med, 44, 275-281, 2016.

[64] Kotfis, K., et al., 'COVID-19: ICU delirium management during SARS-CoV-2 pandemic', Critical Care, 24(1), 1-9, 2020.

[65] Vasilevskis, E.E., et al.," The cost of ICU delirium and coma in the intensive care unit patient', Med Care, 56(10), 890-7, 2018.

[66] Pandharipande, P.P., et al., 'Longterm cognitive impairment after critical illness', $N$ Engl $J$ Med, 369(14), 1306 -16, 2013.

[67] Ely, E.W., 'The ABCDEF bundle: science and philosophy of how ICU liberation serves patients and families', Crit Care Med, 45(2), 321-30, 2017.

[68] Vincent, J.L., et al., "'Comfort and patient-centred care without excessive sedation: the eCASH concept', Intensive Care Med, 42(6), 962 -71, 2016.

[69] Marra, A., et al., 'Delirium monitoring: yes or no? That is the question", Am J Crit Care, 28(2), 127-35, 2019.

[70] Meng, L., et al., 'Intubation and ventilation amid the COVID-19 outbreak: Wuhan's experience,'” Anesthesiology, 132(6), 1317-1332, 2020. 
[71] Pérez, C.A., 'Looking ahead: The risk of neurologic complications due to COVID-19', Neurology: Clinical Practice, 10(4), 371-374, 2020.

[72] Hwang, C., 'Olfactory neuropathy in severe acute respiratory syndrome: report of A case,' Acta Neurologica Taiwanica, 15(1), 26-28, 2016.

[73] Desforges, M., et al., ' 'Human coronaviruses and other respiratory viruses: underestimated opportunistic pathogens of the central nervous system?' Viruses, 12(1), 14, 2019. 